\title{
Pagetoid Dyskeratosis of the Male Genitalia: Case Report and Review
}

\author{
Tyler Werbel ${ }^{1}$, Philip R. Cohen ${ }^{2}$ \\ 1. School of Medicine, University of California, San Diego, San Diego, USA 2. Dermatologist, San Diego Family \\ Dermatology, National City, USA
}

Corresponding author: Tyler Werbel, tylerwerbel@gmail.com

\begin{abstract}
Pagetoid dyskeratosis is a benign incidental pathologic finding that has been reported in many distinct skin lesions on various locations of the body. A man who had pagetoid dyskeratosis within lesions of the penile shaft is described and similar cases of pagetoid dyskeratosis in lesions of the male genitalia are reviewed. The patient was a 26-year-old healthy man who developed several asymptomatic penile papules that were refractory to topical imiquimod 5\% cream and cryotherapy. Snip biopsies were performed and microscopic examination revealed pagetoid dyskeratosis. PubMed was searched for the following terms: cell, clear, dyskeratosis, genitalia, pagetoid, penile, penis, prepuce, scrotum, and shaft. The papers containing these terms and their references were reviewed. Pagetoid dyskeratosis has been observed in lesions on the prepuce and scrotum; this case report now expands the distribution of this finding to the penile shaft. Clinicians and pathologists should be aware of this intriguing potential incidental finding within skin lesions of the male genitalia.
\end{abstract}

Categories: Dermatology, Pathology, Urology

Keywords: cell, clear, dyskeratosis, genitalia, pagetoid, penile, penis, prepuce, scrotum, shaft

\section{Introduction}

Pagetoid dyskeratosis is a benign incidental pathologic feature. It has been observed in several skin lesions [1]. Herein, a man with penile papules that demonstrated pagetoid dyskeratosis is described, and patients with pagetoid dyskeratosis of the male genitalia are summarized.

\section{Case Presentation}

A 26-year-old man with no history of genital dermatoses developed new penile lesions; he was evaluated on several occasions by his primary care physician. The clinical impressions of his lesions included both condyloma acuminata and molluscum contagiosum. On separate occasions, he was treated with either topical imiquimod $5 \%$ cream or cryotherapy with liquid nitrogen. Two months later, he noticed new lesions on his penile shaft and sought medical evaluation by a dermatologist.

Received 05/21/2018

Review began 05/22/2018 Review ended 05/29/2018 Published 06/01/2018

\section{() Copyright 2018}

Werbel et al. This is an open access article distributed under the terms of the Creative Commons Attribution License CC-BY 3.0., which permits unrestricted use, distribution, and reproduction in any medium, provided the original author and source are credited.
Clinical examination showed three 1-2 mm asymptomatic, flesh-colored papules located on the proximal portion of the dorsal penile shaft: proximal, middle, and distal, respectively (Figure 1). The site was cleaned with an alcohol swab, the lesions were circled, and lidocaine $\mathrm{HCl} 1 \%$ with epinephrine 1:100,000 was injected locally. The lesions were elevated with Adson forceps and subsequently removed with Metzenbaum scissors. Hemostasis of the biopsy sites was achieved with the application of $20 \%$ aluminum chloride. The biopsy sites were treated with topical mupirocin $2 \%$ ointment three times daily until the wounds healed.

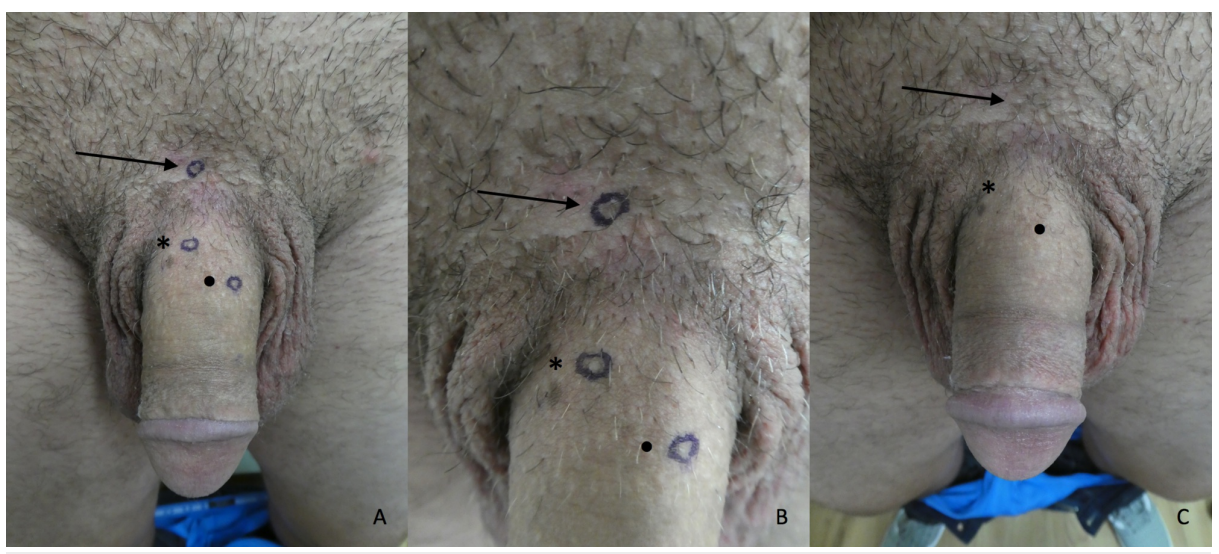

FIGURE 1: Clinical features of pagetoid dyskeratosis 


\section{Cureus}

Distant A) and closer B) views with markings and distant C) view without markings show the clinical features of pagetoid dyskeratosis that presented as proximal (arrow), middle (asterisk), and distal (circle) asymptomatic, flesh-colored papules on the proximal dorsal penile shaft of a 26 -year-old man.

Microscopic examination was performed; hematoxylin and eosin-stained slides of the lesions were inspected with light microscopy. The most proximal lesion revealed focal dermal fibrosis. In addition, there were multiple large, round intraepidermal pale cells presenting singly and in clusters (Figure 2). The cells resembled those seen in extramammary Paget's disease, containing condensed pyknotic nuclei with perinuclear halos of clear cytoplasm. Additionally, they demonstrated premature keratinization without acantholysis or parakeratosis.

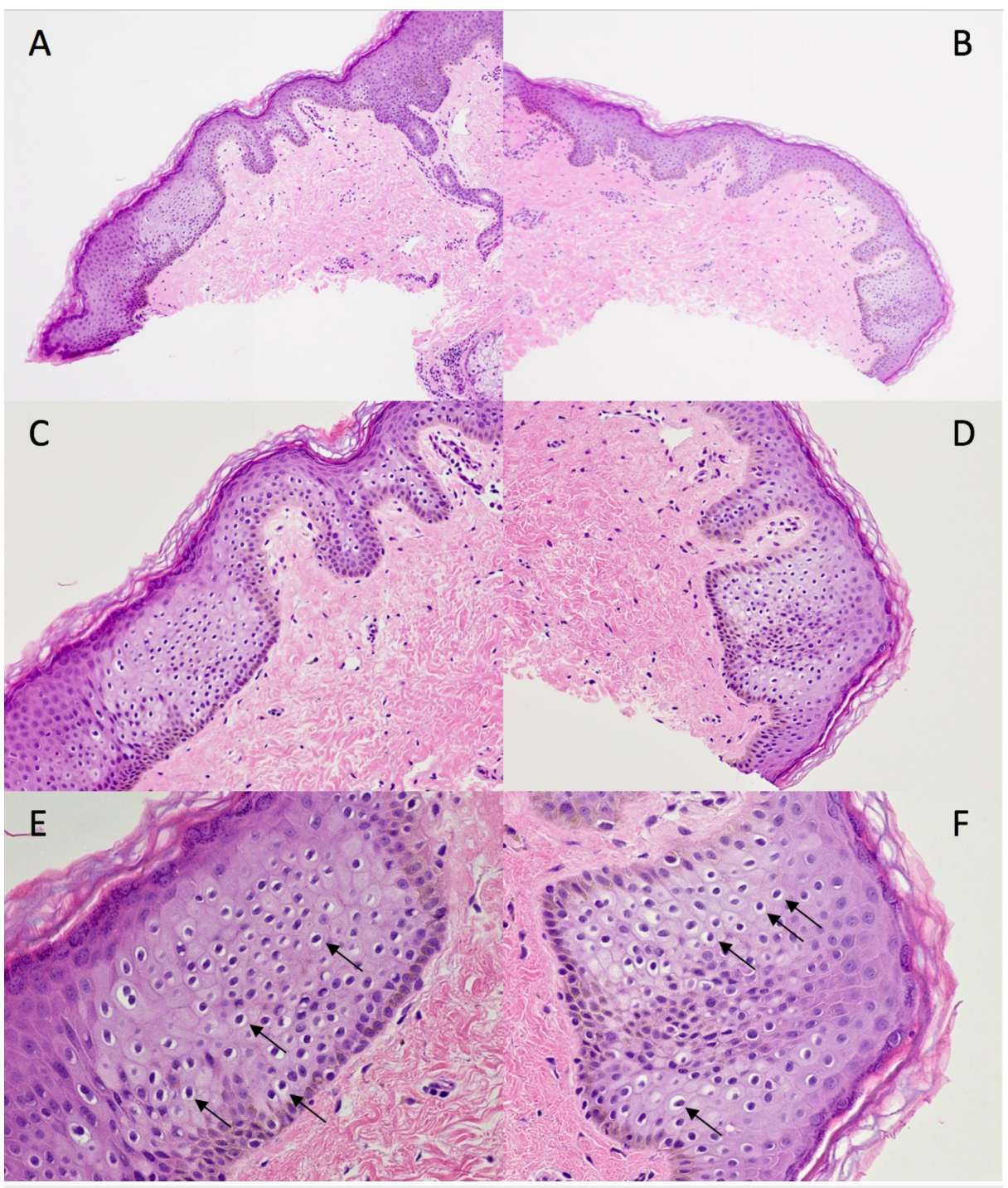

\section{FIGURE 2: Pathology of the proximal papule}

Pathology features of pagetoid dyskeratosis are present in the asymptomatic, flesh-colored papule at the base of the dorsal penile shaft of a 26 -year-old man. There are multiple large, round intraepidermal pale cells extending singly and in clusters upwards in the epidermis. The pagetoid cells (arrows) contain condensed pyknotic nuclei with perinuclear halos of washed out cytoplasm. Additionally, they demonstrated premature keratinization without acantholysis or parakeratosis. In addition, areas of focal dermal fibrosis were also noted. (Hematoxylin and eosin: A: x10; B: x10; C: x20; D: x20; E: x40; F: x40).

The middle lesion revealed a dilated follicular ostium as well as similar changes of pagetoid cells with premature keratinization (Figure 3). The distal lesion only demonstrated sparse superficial dermal fibrosis with mild perifollicular lymphocytic inflammation. The light staining pagetoid cells were not present. Immunoperoxidase staining with p16 was negative within the lesional keratinocytes of all three lesions, making a human papillomavirus infection unlikely. 


\section{Cureus}

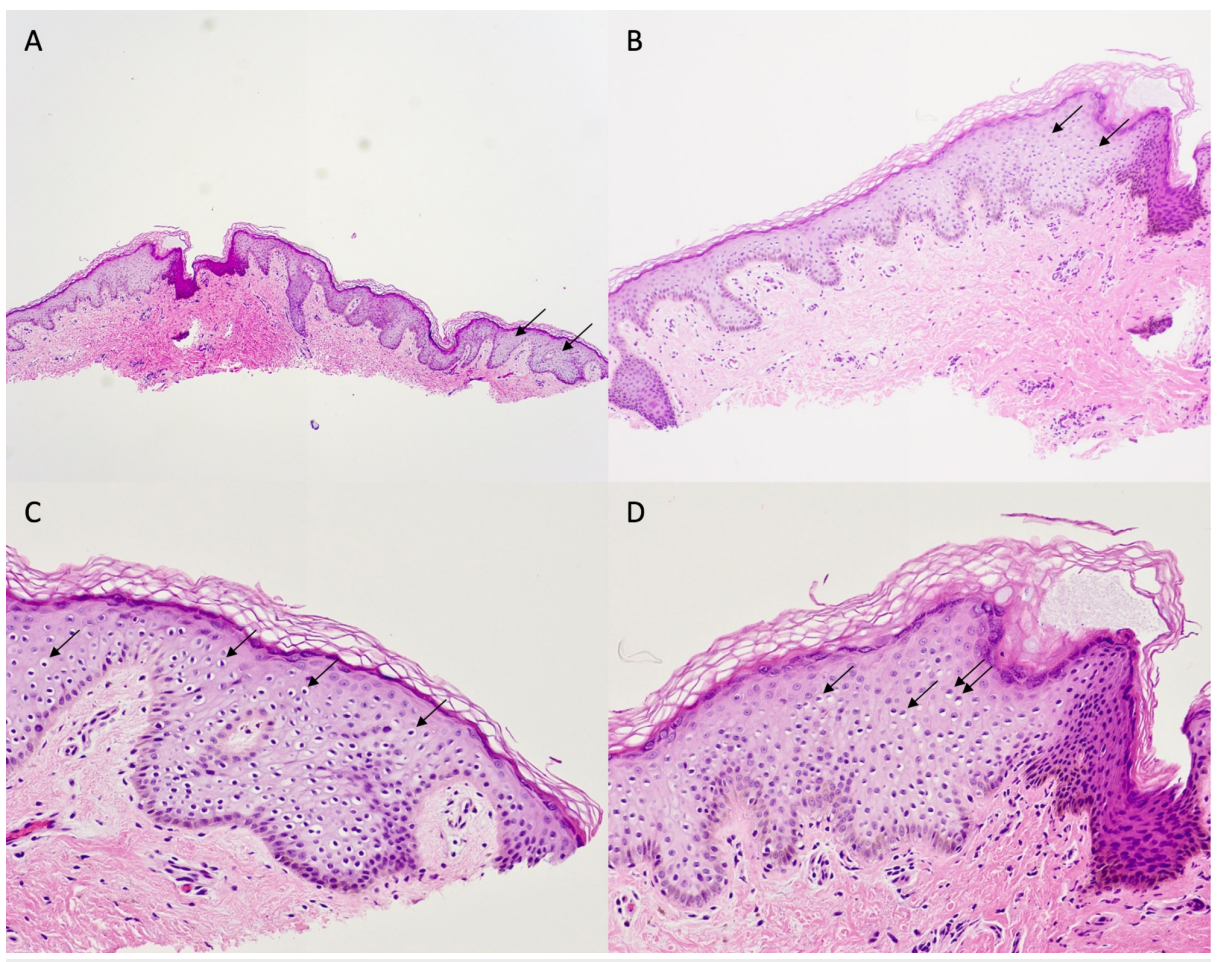

\section{FIGURE 3: Pathology of the middle papule}

Pathology features of another asymptomatic, flesh-colored papule on the proximal dorsal penile shaft of a 26-year-old man also demonstrate pagetoid dyskeratosis. The opening of the hair follicle is dilated. Pagetoid cells (arrows) with premature keratinization are also prominent within the epidermis. (Hematoxylin and eosin: A: $x 4$; B: x10; C: x20; D: x20).

There has been no recurrence or new lesions in the subsequent six months.

\section{Discussion}

Pagetoid dyskeratosis is an incidental pathologic change. It may occur with several different skin conditions. It is characterized by the presence of intraepidermal keratinocytes that resemble those of extramammary Paget's disease [1].

This phenomenon was initially interpreted by Mehregan and Civette as artefactual and potentially being related to intraepidermal anesthesia, occlusion, and moisture, or poor sample fixation [2-3]. However, in 1988, Tschen et al. suggested that it more likely represented a process of induced premature keratinization, terming the entity "pagetoid dyskeratosis" since the cells appear similar to those observed in lesions of extramammary Paget's disease [1]. Subsequently, this pathologic finding has been observed by many other investigators in several distinct anatomic sites and lesions; however, the pathogenesis of pagetoid dyskeratosis remains poorly understood.

The clinical presentation of pagetoid dyskeratosis is variable. It is typically an incidental finding in other primary skin lesions that have been biopsied (Table 1) [1, 4-5]. Hence, the morphology of the lesioncontaining pagetoid dyskeratosis corresponds to that lesion's primary diagnosis. 


\section{Cureus}

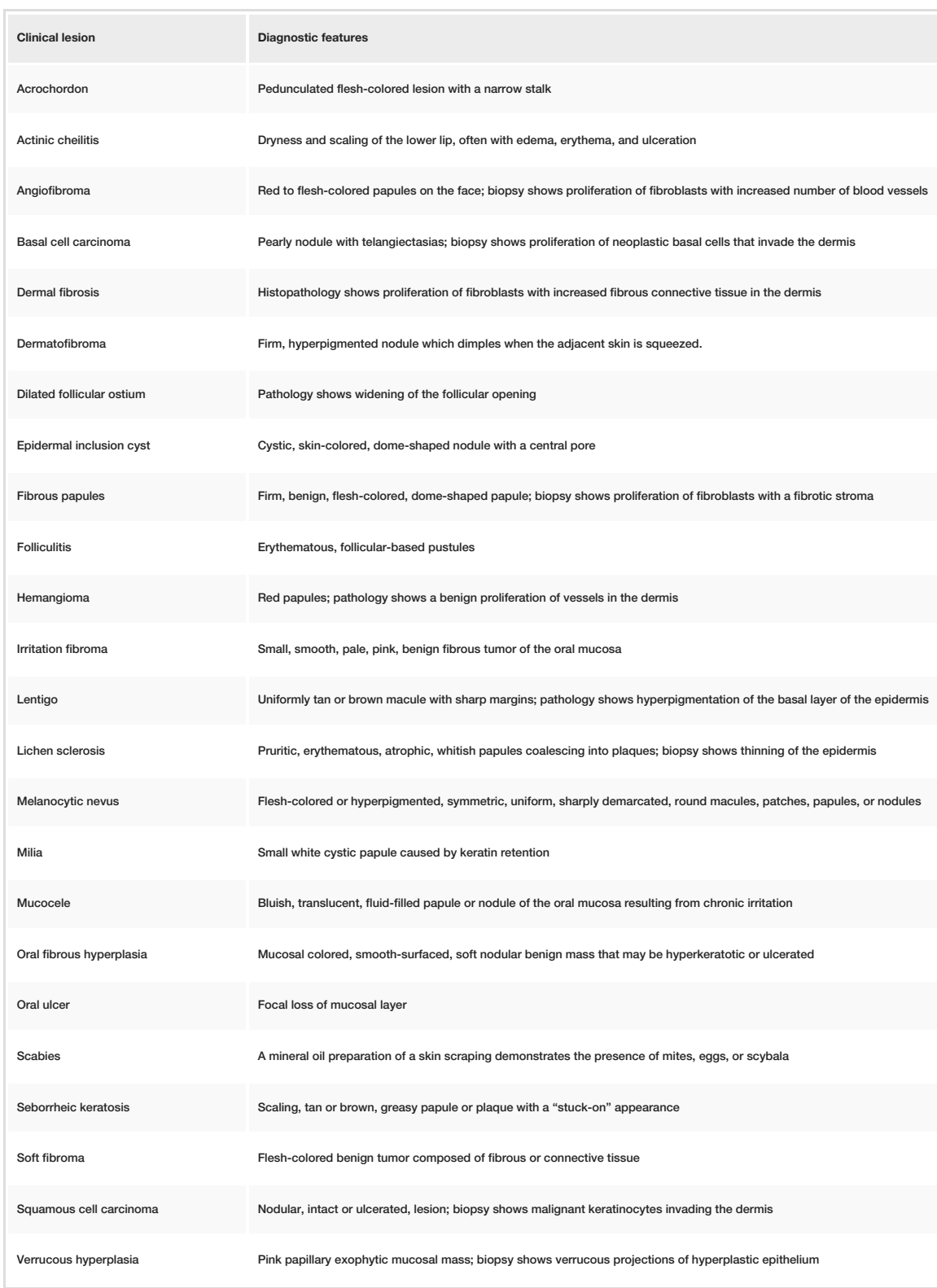

TABLE 1: Clinical Lesions in Which Pagetoid Dyskeratosis Has Been Observed

Pagetoid dyskeratosis is most commonly found in intertriginous areas [1]. However, it can be found nearly anywhere on the body, including the anus (hemorrhoids), buttocks, cervix, extremities, face, hands, nipple, trunk, and vulva [1-2, 5-11]. Pagetoid dyskeratosis of the male genitalia has only been previously described by two other groups of investigators [12-13].

The first study to observe pagetoid dyskeratosis involving the genitalia of men was conducted to examine the histopathology of 281 consecutive patients undergoing circumcision for phimosis. Pagetoid dyskeratosis of the prepuce was incidentally found in 105 individuals (37.4\%). The pagetoid cells were most often seen in the superficial layers of the epidermis but were also occasionally present in the parabasal layer [12].

The second report of male genitalia with pagetoid dyskeratosis included a 54-year-old man with a 10-year history of pruritus involving the scrotum. Cutaneous examination of the scrotum revealed mild erythematous and skin-colored patches with focal areas of hyperpigmentation. Histopathologic analysis was consistent with pagetoid dyskeratosis [13].

Our patient had a history of penile shaft and suprapubic lesions that were clinically assessed to be associated with human papillomavirus or pox virus or both. They were treated topically with either imiquimod or liquid nitrogen cryotherapy and resolved. However, he subsequently developed new small papular lesions affecting the previously treated area of his proximal penile shaft that morphologically was not classic in appearance 


\section{Cureus}

for condyloma acuminatum or molluscum. Indeed, the most prominent feature observed during evaluation of the biopsied lesions was the pagetoid dyskeratosis within the epidermis; the subtle accompanying changes in the dermis (perifollicular inflammation and/or mild dermal fibrosis) correlated with the clinical presentation of the papules.

Microscopically, pagetoid dyskeratosis consists of large round epithelial keratinocytes with pyknotic nuclei and perinuclear halos of washed out cytoplasm. The cells demonstrate premature keratinization into orthokeratotic squames without acantholysis or parakeratosis. They can present individually or in groups often extending upwards in the epidermis. Atypia and mitoses are usually absent [4].

Immunohistochemical staining for high molecular weight cytokeratin and pancytokeratin demonstrates a strong positive signal in the pagetoid cells in comparison to the surrounding keratinocytes [5, 9].

Carcinoembryonic antigen, epithelial membrane antigen, human papillomavirus, and low molecular weight cytokeratin immunohistochemical stains are negative [7]. Staining of pagetoid dyskeratosis cells with alcian blue, Fontana-Masson silver, Mayer's mucicarmine, Mowry's colloidal iron, and periodic-acid Schiff typically yields negative results $[5,7]$.

The pathologic differential diagnosis of pagetoid dyskeratosis includes other conditions with clear cells in the epidermis (Table 2) [3, 14-16]. In some circumstances, immunoperoxidase staining or other stains (in addition to hematoxylin and eosin) may help differentiate these conditions. In our patient, the possibility of condyloma acuminatum was excluded by negative expression of the cells after p16 staining. 


\section{Cureus}

\begin{tabular}{|c|c|}
\hline Pathologic differential diagnosis & Differentiating features \\
\hline Balloon cell melanoma & Hyperchromatic nuclei surrounded by abundant vacuolated cytoplasm, nests of melanocytes at the DEJ, + S100 \\
\hline Clear cell acanthoma & Erythematous, sharply demarcated, solitary papule on the lower extremities; basal layer intact, + PAS \\
\hline $\begin{array}{l}\text { Clear cell Bowen's disease (squamous cell carcinoma in } \\
\text { situ) }\end{array}$ & Well-demarcated, erythematous, irregularly bordered plaque with crust or scale; atypia, large nuclei, mitoses, no perinuclear halo, intact basal membrane \\
\hline Clear cell eccrine carcinoma & $\begin{array}{l}\text { Rapidly growing, multinodular dermal neoplasm; ductal differentiation and intracytoplasmic lumen formation, mitoses, prominent nucleoli, + low weight CK, + CEA, + } \\
\text { PAS }\end{array}$ \\
\hline Clear cell papulosis & White macules and papules distributed along the milk lines; + mucicarmine, + CEA \\
\hline Clear cell squamous carcinoma & Hydropic degeneration of neoplastic cells, invasion of basal layer, - mucicarmine, - PAS \\
\hline Clear cell syringoma & Association with diabetes mellitus; nests of eccrine ducts, tadpole-like structures in a fibrous stroma, + PAS \\
\hline Condyloma acuminatum & Papillated, smooth or soft anogenital papules or plaques; + p16 \\
\hline Extramammary Paget's disease & Pruritic eczematous, well-demarcated plaque, most often on the vulva; + CK7, + PAS \\
\hline Renal cell carcinoma & Flank mass, hematuria, paraneoplastic syndromes; lipid and glycogen-rich cytoplasm \\
\hline Sebaceous adenoma & Benign proliferation of sebaceous cells \\
\hline Sebaceous carcinoma & Eyelid lesion; atypia, mix of undifferentiated and sebaceous cells, scalloping of the nuclei \\
\hline Sebaceous epithelioma & Malignant proliferation of irregularly shaped cells, half of which demonstrate sebaceous differentiation \\
\hline Superficial spreading malignant melanoma & egularly bordered, multi-colored, pigmented plaque; atypical, hyperchromatic, neoplastic melanocytes that lack cellular maturation, + S100, + HMB45 \\
\hline Toker cell of the nipple & Found in the nipple epidermis of some normal women; - mucicarmine, + CK7 \\
\hline Tricholemmal carcinoma & Atypias, mitoses, + PAS \\
\hline Tricholemmoma & ar growth of clear cells around hair follicles, peripheral palisading, + PA \\
\hline
\end{tabular}

TABLE 2: Pathologic Differential Diagnosis of Pagetoid Dyskeratosis

CD: cluster of differentiation; CEA: carcinoembryonic antigen; CK: cytokeratin; DEJ: dermal-epidermal junction; EMA: epithelial membrane antigen; HMB: human melanoma black; PAS: periodic acid Schiff; -: negative; +: positive

The pathogenesis of pagetoid dyskeratosis remains to be definitively established. Tschen et al. hypothesized that the cells represent a small population of the normal keratinocytes that are induced to proliferate by an external trigger, such as mechanical trauma or friction [1]. Piqué-Duran et al. found that lesions of the axilla more frequently demonstrated pagetoid dyskeratosis in comparison to those of other locations; they suggested that this observation supports the theory that moisture and friction contribute to the development of pagetoid dyskeratosis [14].

Pagetoid dyskeratosis is an incidental finding. Typically, there are no clinical sequelae. Therefore, management is directed toward the primary lesion.

\section{Conclusions}


Pagetoid dyskeratosis is an intriguing benign pathologic feature. It has been observed in a variety of cutaneous lesions located on various areas of the body. Pagetoid dyskeratosis of the male genitalia is uncommon and has been previously described on either the scrotum or the prepuce; our patient's lesions were on the proximal penile shaft. The differential diagnosis of pagetoid dyskeratosis includes other conditions characterized by clear cells in the epidermis. In particular, in men with genital lesions, the differential diagnosis includes venereal warts. This was excluded based on microscopic findings and negative p16 staining. Pagetoid dyskeratosis-directed therapy is usually not necessary and management of the patient is based upon treating the primary skin lesion.

\section{Additional Information}

\section{Disclosures}

Human subjects: Consent was obtained by all participants in this study. Conflicts of interest: In compliance with the ICMJE uniform disclosure form, all authors declare the following: Payment/services info: All authors have declared that no financial support was received from any organization for the submitted work. Financial relationships: All authors have declared that they have no financial relationships at present or within the previous three years with any organizations that might have an interest in the submitted work. Other relationships: All authors have declared that there are no other relationships or activities that could appear to have influenced the submitted work.

\section{References}

1. Tschen JA, McGavran MH, Kettler AH: Pagetoid dyskeratosis: a selective keratinocytic response . J Am Acad Dermatol. 1988, 19:891-94. 10.1016/S0190-9622(88)70250-9

2. Mehregan AH: Clear epidermal cells: an artifact . J Cutan Pathol. 1980, 7:154-58. 10.1111/j.16000560.1980.tb01194.x

3. Civatte J: Clear-cell tumors of the skin: a histopathologic review . J Cutan Pathol. 1984, 11:165-75. 10.1111/j.1600-0560.1984.tb00369.x

4. Santos-Briz A, Cañueto J, del Carmen S, et al.: Pagetoid dyskeratosis in dermatopathology. Am J Dermatopathol. 2015, 37:261-65. 10.1097/DAD.0000000000000284

5. Garijo MF, Val D, Val-Bernal JF: Pagetoid dyskeratosis of the lips. Am J Dermatopathol. 2001, 23:329-33. 10.1097/00000372-200108000-00010

6. Garijo MF, Val D, Val-Bernal JF: Pagetoid dyskeratosis of the nipple epidermis: an incidental finding mimicking Paget's disease of the nipple. APMIS. 2008, 116:139-46. 10.1111/i.1600-0463.2008.00826.x

7. Val-Bernal JF, Pinto J, Garijo MF, Gómez MS: Pagetoid dyskeratosis of the cervix: an incidental histologic finding in uterine prolapse. Am J Surg Pathol. 2000, 24:1518-23. 10.1097/00000478-200011000-00007

8. Val-Bernal JF, Pinto J: Pagetoid dyskeratosis is a frequent incidental finding in hemorrhoidal disease . Arch Pathol Lab Med. 2001, 125:1058-62.

9. Val-Bernal JF, Diego C, Rodriguez-Villar D, Garijo MF: The nipple-areola complex epidermis: a prospective systematic study in adult autopsies. Am J Dermatopathol. 2010, 32:787-93. 10.1097/DAD.0b013e3181ddbec5

10. Salphale P, Thomas M: Pagetoid dyskeratosis of the forehead. Indian Dermatol Online J. 2014, 5:215-16. 10.4103/2229-5178.131128

11. Wang LC, Medenica MM, Shea CR, Busbey S: Pagetoid dyskeratosis of the hand. J Am Acad Dermatol. 2004, 50:483-84. 10.1016/S0190-9622(03)02109-1

12. Val-Bernal JF, Garijo MF: Pagetoid dyskeratosis of the prepuce. An incidental histologic finding resembling extramammary Paget's disease. J Cutan Pathol. 2000, 27:387-91. 10.1034/j.1600-0560.2000.027008387.x

13. Lee HR, Han TY, Lee JH, Son SJ: Pagetoid dyskeratosis of the scrotum: histologic findings resembling extramammary Paget's disease. Am J Dermatopathol. 2011, 33:755-57. 10.1097/DAD.0b013e31820a264f

14. Piqué-Duran E, Palacios-Llopis S, Moreno-Ramis P, et al.: Comparative study of pagetoid dyskeratosis between acrochordons and soft fibromas. Am J Dermatopathol. 2006, 28:478-81. 10.1097/01.dad.0000208265.05109.a0

15. Biswas A, Mahalingam M: Cutaneous clear cell neoplasms: a histopathological reappraisal. Am J Dermatopathol. 2012, 34:237-54. 10.1097/DAD.0b013e3182106d28

16. Suster S: Clear cell tumors of the skin. Semin Diagn Pathol. 1996, 13:40-59. 